\title{
Feasibility study of internal conversion electron spectroscopy of ${ }^{229 m} \mathrm{Th}$
}

\author{
Benedict Seiferle ${ }^{1, a}$, Lars von der Wense $^{1}$, and Peter G. Thirolf ${ }^{1}$ \\ Ludwig-Maximilians-Universität München, Am Coulombwall 1, Garching, Germany
}

Received: 1 February 2017 / Revised: 12 April 2017

Published online: 25 May 2017

(c) The Author(s) 2017. This article is published with open access at Springerlink.com

Communicated by A. Jokinen

\begin{abstract}
With an expected energy of $7.8(5) \mathrm{eV}$, the isomeric first excited state in ${ }^{229} \mathrm{Th}$ exhibits the lowest excitation energy of all known nuclei. Until today, a value for the excitation energy has been inferred only by indirect measurements. In this paper we propose an experimental method that is potentially capable of measuring the ground-state transition energy via the detection of the internal conversion electrons. MatLabbased Monte Carlo simulations have been performed to obtain an estimate of the expected statistics and to test the feasibility and the expected precision of the experiment. From the simulations we conclude that with the presented methods an energy determination with a precision of better than $0.1 \mathrm{eV}$ is within reach.
\end{abstract}

\section{Introduction}

The isomeric first excited state of ${ }^{229} \mathrm{Th}\left({ }^{229 m} \mathrm{Th}\right)$, is subject to current vivid research.

Among all known nuclear excited states it is the only one that could allow for a direct optical laser excitation, due to its extraordinary low excitation energy of only $7.8(5) \mathrm{eV}$, corresponding to about $160 \mathrm{~nm}[1,2]$. This has led to a multitude of proposals for possible applications, including a nuclear optical clock $[3,4]$ that could provide a complementary technology to today's existing optical atomic clocks, potentially even outperforming the present frequency standards due to the superior resilience of a nuclear clock against external perturbations. It took 40 years until the first direct identification of the ground-state decay of ${ }^{229 m}$ Th via the observation of its internal conversion decay branch [5]. However, despite large experimental efforts conducted worldwide [6-13], the uncertainty in the excitation energy value is still too large to allow for a direct laser excitation. By now, energy values have only been obtained by indirect measurements, investigating nuclear excited states at higher energies and $\gamma$ rays emitted in their decays to the ground and isomeric state $[1,2,14-16]$.

There are three decay channels of ${ }^{229 m}$ Th to its ground state discussed in the literature $[17,18]$ : i) internal conversion (IC), which proceeds via the emission of an electron with an energy of $E_{e}=E_{I}-E_{B}$, where $E_{I}$ is the isomeric energy with respect to the ground state and $E_{B}$ is the binding energy of the electron. ii) $\gamma$ decay, where the emitted photon carries the energy of the isomer, and

\footnotetext{
a e-mail: benedict.seiferle@physik.uni-muenchen.de
}

iii) bound internal conversion, which proceeds via the excitation of a bound electronic shell state.

While a variety of proposals and experimental attempts can be found in the literature, aiming at the direct measurement of a VUV photon emitted during the ground-state decay of ${ }^{229 m}$ Th $[6,7,19]$, this paper investigates the possibilities of an energy determination via the electron that is emitted during the already experimentally observed internal conversion decay $[5,20]$.

Internal conversion electron spectroscopy of ${ }^{229 m} \mathrm{Th}$ has several advantages compared to the photonic approach: due to the large conversion coefficient, the decay via internal conversion is about $10^{9}$ times faster than the photon decay. Therefore it is possible to trigger the IC decay by neutralizing a ${ }^{229 m}$ Th ion: IC decay is only possible if the binding energy of an electron in the surrounding of the nucleus is below the isomeric energy. Therefore IC is suppressed in ${ }^{229 m} \mathrm{Th}$ ions, but not in neutral thorium atoms [21]. In the experimental approach it is assumed that the isomer decays via internal conversion on the surface of a solid. The energy of the isomer is transferred to the bound surface electrons which are thereby able to leave the surface. In the following, measurement principles are discussed to check the feasibility of high-precision IC electron spectroscopy of the ${ }^{229} \mathrm{Th}$ isomer.

\section{Simulated setup}

In our experimental setup, ${ }^{229} \mathrm{Th}$ ions are produced as $\alpha$ recoil ions from a thin extended ${ }^{233} \mathrm{U}$ source, where a decay branch of $2 \%$ ends up in the isomeric first excited state 

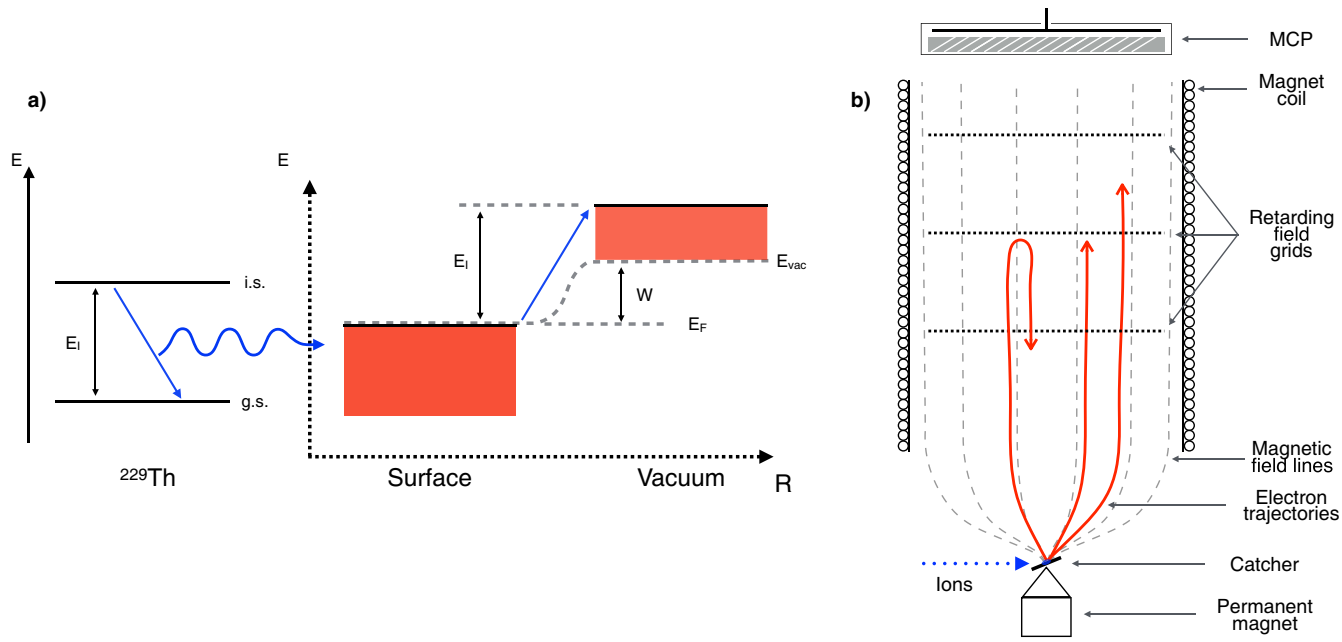

Fig. 1. (a) Illustration of the energy levels of the ground state (g.s.) and first isomeric excited state (i.s.) in the ${ }^{229} \mathrm{Th}$ nucleus as well as the energy levels of electrons in the surface. When the excited state decays, its energy is transferred to the electrons in the sample surface, which are thereby ejected into vacuum. Here $W$ denotes the work function, $E_{\mathrm{vac}}$ the local vacuum energy, $E_{F}$ the Fermi energy and $E_{I}$ the energy of the isomeric state with respect to the ground state. (b) Sectional view of the envisaged setup. ${ }^{229(\mathrm{~m})} \mathrm{Th}$ ions are entering from the left and are collected on a catcher placed on the central axis of a magnetic bottle spectrometer. Potentially emitted IC electrons are guided by the magnetic field lines and their energy is analyzed with a retarding field analyzer.

of ${ }^{229} \mathrm{Th}$. In the following, the notation ${ }^{229(\mathrm{~m})}$ Th refers to an ensemble of ions containing the ground state and the isomeric first excited state. ${ }^{229(m)}$ Th ions are stopped in a buffer-gas stopping cell filled with ultra-pure helium, so that after thermalization of the $\alpha$-recoil ions in the buffer gas and their transport via RF and DC fields to an extraction nozzle ${ }^{229(m)} \mathrm{Th}$ ions can be extracted into a segmented radio frequency quadrupole (RFQ) ion guide and phase-space cooler structure. The segmented structure of the RFQ allows to form ion bunches. A subsequent quadrupole mass separator removes the accompanying $\alpha$ decay daughter products. A detailed study of the experimental setup can be found in [22]. A width of the ion bunches of $10 \mu \mathrm{s}\left(\mathrm{FWHM}_{\mathrm{TOF}}\right)$, with $\approx 200^{229(m)} \mathrm{Th}^{3+/ 2+}$ ions per bunch was achieved at a rate of $10 \mathrm{~Hz}$ [20]. The ion bunches contain ${ }^{229 m} \mathrm{Th}$, which has already been detected with this setup [5].

The general idea of performing internal conversion electron spectroscopy of ${ }^{229 m} \mathrm{Th}$ is to guide the extracted, mass-separated and bunched ions towards an electron spectrometer, where they will be neutralized, thereby triggering the IC decay and to measure the subsequently emitted electrons, see fig. 1.

In this work an approach is proposed where ${ }^{229 m} \mathrm{Th}$ ions are collected directly on a metallic catcher for neutralization. The electrons in the solid are dislocated, therefore it must be expected that ${ }^{229 m} \mathrm{Th}$ decays via internal conversion by emitting an electron from the surface. The electron spectrum must reflect the electrons binding energies in the surface. As there are only about 4 ions in the isomeric state extracted per bunch, it is advantageous to use a spectrometer with a high efficiency. Therefore, a magnetic bottle-type spectrometer [23], providing an acceptance angle of nearly $4 \pi$, is envisaged (see fig. $1(\mathrm{~b})$ ). In such a spectrometer, electrons are collected and collimated by a magnetic gradient field. In general, the electron energy is then either inferred by a time-of-flight method or by retarding fields. In case of ${ }^{229 m} \mathrm{Th}$, the time-of-flight method cannot be used, since the lifetime of the internal conversion decay is roughly $10 \mu \mathrm{s}$ and thus long compared to the short flight time of electrons $\left(v \approx 6 \times 10^{5} \mathrm{~m} / \mathrm{s}\right.$ for $1 \mathrm{eV}$ electrons). To achieve $10 \%$ energy resolution for $1 \mathrm{eV}$ electrons, one would need a drift tube with a length of $\approx 40 \mathrm{~m}$ to compensate the $10 \mu \mathrm{s}$ IC lifetime. By applying retarding fields with high-transmission grids an integrated spectrum is generated where all electrons are counted, whose energy is sufficient to pass the retarding fields.

\subsection{Solid sample and surface effects}

A simple way to neutralize the ${ }^{229 m} \mathrm{Th}$ ions is to collect them on a metallic catcher. If the implantation depth is not too large ${ }^{1}$, electrons emitted during the decay should be able to leave the sample and be measured by the spectrometer. The inelastic mean free path of an electron $(\lambda)$ in a metal with a kinetic energy of $E_{\text {kin }}<5 \mathrm{eV}$ is $\lambda>6 \mathrm{~nm}[25]$. A possible source of background for experiments with slow ions is Auger electron emission (see [26]). When ion bunches are used, these electrons can be distinguished from electrons emitted by the isomeric decay: due to its lifetime of $\approx 10 \mu \mathrm{s}$, the isomeric decay can be separated in time from signals potentially generated by the ionic impact [20].

\footnotetext{
1 The stopping range for thorium ions with $E_{\text {kin }}=100 \mathrm{eV}$ $(500 \mathrm{eV})$ in gold is $5 \AA$ ( $8 \AA$ ) (values are taken from SRIM simulations [24]).
} 

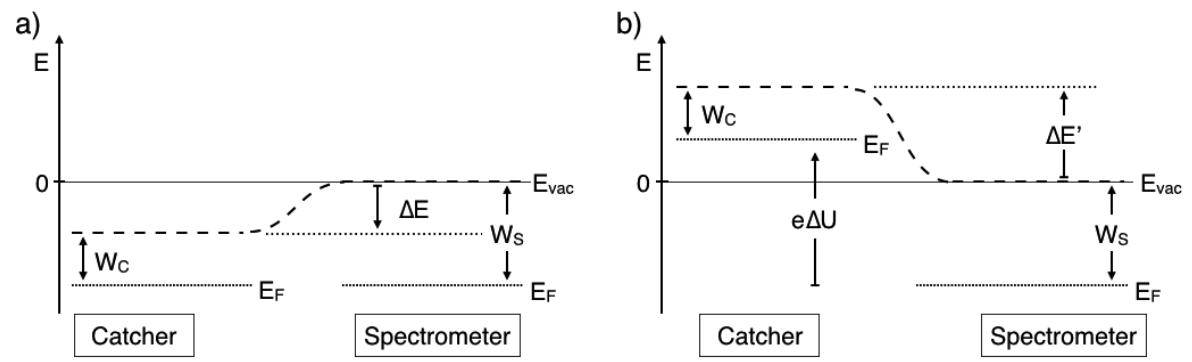

Fig. 2. Visualization of the work function of the catcher and spectrometer (see also [30]). $W_{C}$ denotes the catcher work function, $W_{S}$ the spectrometer work function, $E_{F}$ the Fermi energy and $E_{\mathrm{vac}}$ the local vacuum energy levels. Two situations are shown: (a) a contact potential difference $\Delta E$ generated between the catcher and the spectrometer; (b) the influence of an applied offset voltage $\Delta U$ on the contact potential difference.

It can be expected that the energy distribution of the electrons reflects the electronic structure of the catcher's surface. The processes are similar to metastable atom electron spectroscopy (MAES) $[27,28]$, that is used to study the electronic structure of surfaces.

\subsubsection{Surface influence}

In this section, the influence of the catcher material (or sample) is investigated and a possible measurement scheme is shown.

The problem of measuring the isomer's energy is similar, but not identical to ultraviolet photo electron spectroscopy, where a surface is irradiated with UV photons of known energy [29]. The electronic structure is then inferred from the energy of electrons emitted during the photoelectric effect. In contrast to ultraviolet photo electron spectroscopy, when measuring the isomeric energy, it is the objective to infer the energy of the "light source" (i.e., isomer) from the energy distribution of the electrons. In the following, a short review of the terms and measurement schemes deployed in photo electron spectroscopy is given (see also [30]):

The work function $W$ of a metallic material is defined as the potential energy difference between the local vacuum level $\left(E_{\mathrm{vac}}\right)$ and the Fermi level $\left(E_{F}\right)$ :

$$
W=E_{\mathrm{vac}}-E_{F} .
$$

When two materials (for example the spectrometer and the catcher surface with work functions $W_{S}$ and $W_{C}$ ) are in electrical contact, their Fermi levels align. If their work functions differ, a potential difference between the local vacuum levels is generated. The contact potential difference amounts to

$$
\Delta E=W_{C}-W_{S} .
$$

In our situation, a contact potential difference may be generated between the catcher and the spectrometer, which is visualized in fig. 2(a). Therefore, if the work function of the spectrometer exceeds the catcher's work function, an offset voltage $\Delta U$ needs to be applied to the sample in order to give the electrons enough energy to overcome the contact potential difference. Consequently, the contact potential difference is shifted by $\Delta U$ (see fig. 2(b)):

$$
\Delta E^{\prime}=W_{C}-W_{S}+e \Delta U .
$$

Photons of energy $h \nu$ may eject electrons from a metallic surface with a work function $W_{C}$, as long as $h \nu \geq W_{C}$. The energy of such a photo electron is described by a Fermi distribution with a maximum energy of

$$
E_{C}^{\max }=h \nu-W_{C} .
$$

Note that in this definition $E_{C}^{\max }$ is given with respect to the local vacuum energy level of the catcher. Given the shifted contact potential difference with the spectrometer, $\Delta E^{\prime}$, the maximum kinetic energy of the electrons measured with the spectrometer amounts to

$$
\begin{aligned}
E_{S}^{\max } & =h \nu-W_{C}+\Delta E^{\prime} \\
& =h \nu-W_{C}+W_{C}-W_{S}+e \Delta U \\
& =h \nu-W_{S}+e \Delta U .
\end{aligned}
$$

From the above equation it is obvious that the energy of the electrons in the end does not depend on the value of the work function of the catcher, but only on the spectrometer work function and the applied offset voltage $\Delta U$. Treating the isomeric decay of ${ }^{229 m} \mathrm{Th}$ as a photon with energy $E_{I}=h \nu$ that is coupling to the electrons in the catcher surface, the energy of the isomer $E_{I}$ can be inferred by the following equation:

$$
E_{I}=E_{S}^{\max }+\left(W_{S}-e \Delta U\right),
$$

where the expression $\left(W_{S}-e \Delta U\right)$ can be measured with a light source of known energy and using eq. (7). Therefore, the only remaining surface influence of the sample on the maximum kinetic energy of an electron is the temperaturedependent Fermi distribution of $E_{e}^{\max }$, but not the value of the sample work function.

\section{Simulations}

In order to get an estimate for the count rates and resulting integrated spectra that can be measured for ${ }^{229 m} \mathrm{Th}$ IC 
Table 1. List of input values that were used for the simulations as discussed in the text and shown in the plots (figs. 3, 4 and 5). The read-out time is the width of the time window in which counts of the isomer are expected and read out.

\begin{tabular}{lr|lr|cc}
\hline Spectrometer efficiency & $\%$ & \multicolumn{3}{|c}{ Simulation input } \\
\hline Collimation & 80 & MCP dark count rate $[1 / \mathrm{s}]$ & 35 & Collection efficiency $[\%]$ & 20 \\
Grid transmission & 50 & Bunches per second $[1 / \mathrm{s}]$ & 10 & Resolution $(\mathrm{FWHM})[\mathrm{eV}]$ & 0.1 \\
MCP detection & 30 & Ions per bunch & 200 & $T[\mathrm{~K}]$ & 300 \\
Hemisphere & 50 & Read out time per bunch $[\mu \mathrm{s}]$ & 200 & & \\
\hline Total spectrometer efficiency: & 6 & & & \\
\hline
\end{tabular}

a)

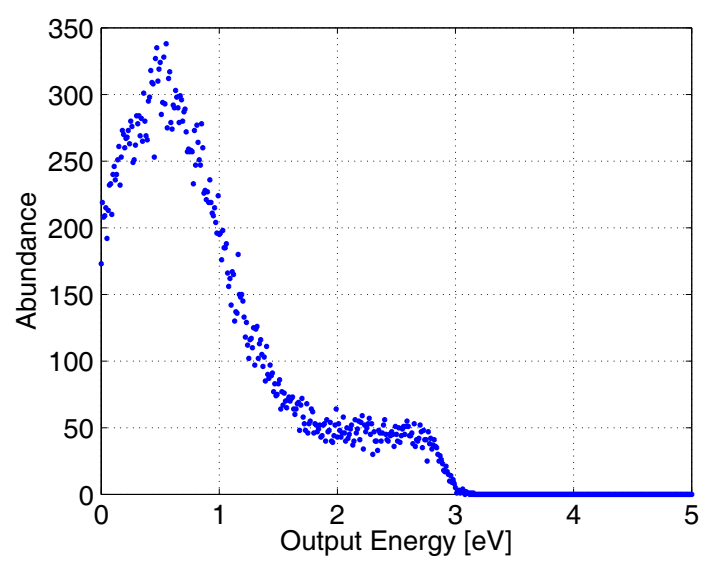

b)

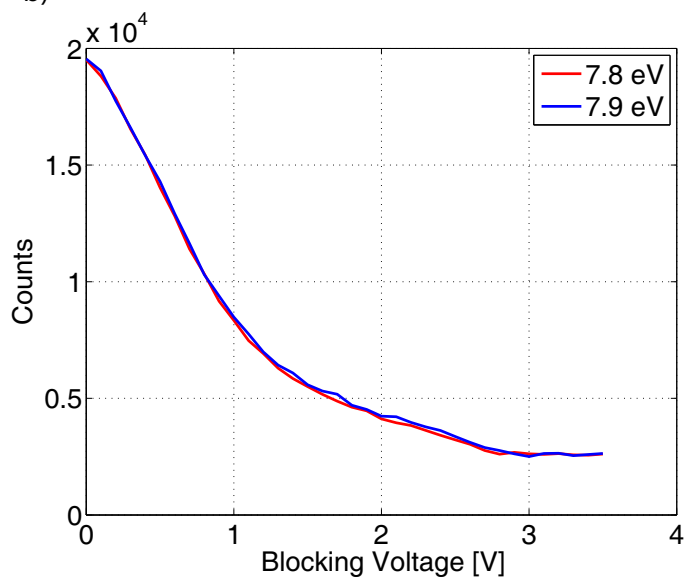

Fig. 3. Simulated spectra: (a) the simulated energy distributions for a "bare" Fermi distribution with some arbitrary additional underlying electronic structure. (b) Corresponding results from a simulated measurement with the retarding field spectrometer. A measurement time of $360 \mathrm{~h}$ and a blocking voltage increment of $0.1 \mathrm{~V}$ was chosen.

electrons emitted from a solid sample, Monte Carlo (MC) simulations were performed with a custom MC code. The code allows for sampling a pseudo-random number generated from an arbitrary distribution [31] and is used to simulate a predefined kinetic energy distribution $D(E)$ of the electrons.

\subsection{Description of the simulation process}

The number of isomers per bunch is calculated by taking the $2 \%$ branching ratio to the isomeric state from the ${ }^{233} \mathrm{U} \alpha$ decay for the 200 ions contained in one bunch. In this way, we are left with 4 ions in the isomeric state per bunch. We further assume that only $20 \%$ of the ions are collected in the center of the spectrometer and contribute to a spectrum. A collimation efficiency of the magnetic field of $80 \%$, a combined grid transmission of $50 \%$ (3 grids with $80 \%$ geometrical transmission each) and a detection efficiency of $30 \%$ was used. Since a catcher surface is used, only $50 \%$ of the IC electrons that are potentially emitted in one hemisphere can be collected. In this way we are left with a total combined detection efficiency of the spectrometer of $\epsilon=6 \%$. General input values that were used for the simulation, such as the detection properties, count rates, resolution and temperature are listed in table 1 . The background was simulated by calculating the signal-to-background ratio and simulating the dark counts accordingly.

As already mentioned in sect. 2.1.1, the maximum energy of the electrons (with respect to the spectrometer) does not depend on the work function of the sample, but rather on the work function of the spectrometer and the sample offset voltage $\left(W_{S}-e \Delta U\right)$, that needs to be obtained from a calibration measurement with a light source of known energy. Nevertheless, the work function of the sample does play a role, since $h \nu=E_{I} \geq W_{C}$ must always be satisfied and the electron energies (with respect to the vacuum level of the sample) are distributed between 0 and $\left(E_{I}-W_{C}\right) \mathrm{eV}$. As a typical work function of metals, $W_{C}$ was set to $5 \mathrm{eV}$. In the simulations $\left(W_{S}-e \Delta U\right)$ was set to be equal to $5 \mathrm{eV}$ (the offset voltage was chosen to balance the contact potential difference). In this way, the electron energies are distributed over a range between 0 and $E_{I}-5 \mathrm{eV}$. For the isomer energy $E_{I}$ two values were simulated: $7.8 \mathrm{eV}$ and $7.9 \mathrm{eV}$. The two values with a difference of $0.1 \mathrm{eV}$ were chosen in order to check the resolving power of this approach. Since the photoelectrons reflect the surface's electronic structure, one cannot assume a "bare" Fermi distribution in the simulation. This is taken into account by adding a Gaussian energy distribution to the low-energy part of the electron spectrum, so that only $\approx 10 \%$ of the electrons have a higher energy than $2 \mathrm{eV}$. The simulated energy distribution and resulting spectra are shown in fig. 3 . 
a)

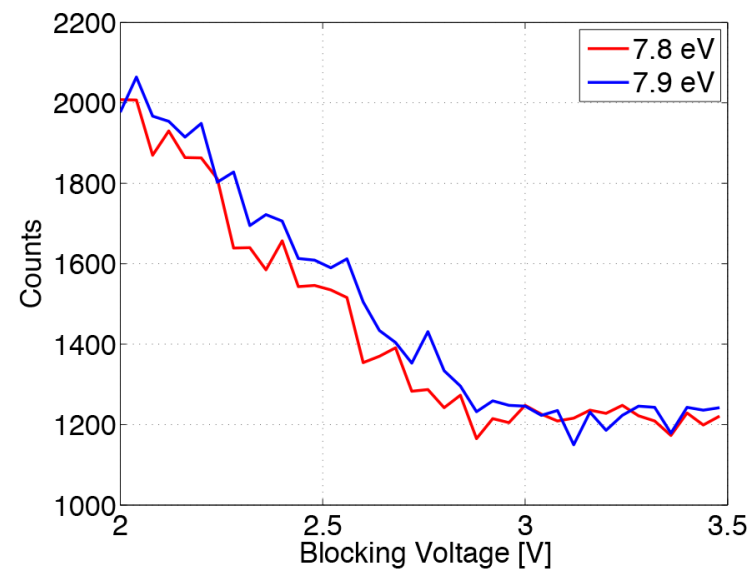

b)

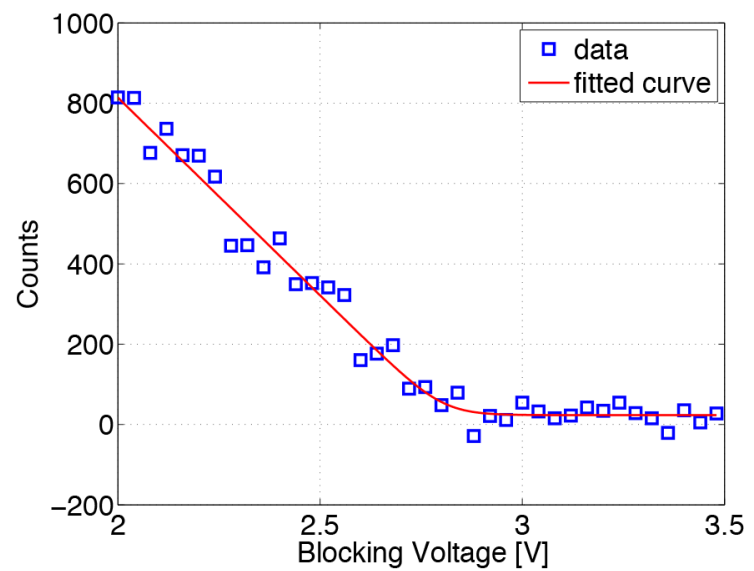

Fig. 4. (a) Simulated spectra for two isomeric energies $(7.8 \mathrm{eV}$ and $7.9 \mathrm{eV})$ with a measurement time of $180 \mathrm{~h}$, blocking voltage increment of $0.04 \mathrm{~V}$ in a range of $1.5 \mathrm{~V}$ (leading to $4.8 \mathrm{~h}$ measurement time per data point), $W_{C}=5 \mathrm{eV}$ and $\left(W_{S}-\Delta U e\right)=5 \mathrm{eV}$. (b) One of the fitted curves (according to eq. (10)). The fit values were $E=2.80 \pm 0.05 \mathrm{eV}$ (for $7.8 \mathrm{eV}$ ) and $E=2.91 \pm 0.05 \mathrm{eV}$ (for $7.9 \mathrm{eV}$ ). Note that the calculated background (1210 counts) was subtracted in the fit plot.

\section{Analysis of simulated spectra}

Spectra measured with retarding field analyzers are typically differentiated to gain information on the electronic structure of the surfaces. Since we are only interested in the maximum energy of the electron (i.e., the Fermi edge) and a differentiation may lead to large relative errors, we directly fit the indefinite integral of the Fermi function to the high-energy part of the integrated spectrum.

When a solid sample is used for the neutralization of the ${ }^{229 m}$ Th ions, the subsequently emitted electrons reflect the electronic structure of the surface (see sect. 2.1.1). Especially, the maximum energy edge must reflect the Fermi distribution:

$$
f(E)=\frac{a}{e^{\left(\left(E-E_{0}\right) / b\right)}+1},
$$

with $b=k_{B} \cdot T, E_{0}$ as the maximum kinetic energy of the electrons and $a$ as a constant. Its definite integral from 0 to $E$ reads

$$
F(E)=a \cdot\left(b \cdot \ln \left[\frac{e^{\left(\left(E-E_{0}\right) / b\right)}+1}{e^{-E_{0} / b}+1}\right]-E\right)+C,
$$

where $C$ is a constant. Figure 4 shows the simulation of the measurements described above with voltage increments of $0.04 \mathrm{~V}$ over a range of $1.5 \mathrm{~V}$ and a measurement time of $180 \mathrm{~h}$ (leading to 38 data points, with $4.8 \mathrm{~h}$ measurement time per data point) and the corresponding fit plots. For $7.8 \mathrm{eV}(7.9 \mathrm{eV})$ isomeric energy, a maximum kinetic energy of $E_{S}^{\max }=2.80 \pm 0.05 \mathrm{eV}\left(E_{S}^{\max }=2.91 \pm 0.05 \mathrm{eV}\right)$ was obtained from the fit. The isomer's energy is then recovered by using eq. (8), leading to $E_{I}=7.80 \pm 0.05 \mathrm{eV}$ $\left(E_{I}=7.91 \pm 0.05 \mathrm{eV}\right)$.

\section{Energy resolution}

The precision and accuracy of the fit method was probed, by performing 1000 simulations (each with a specific mea-

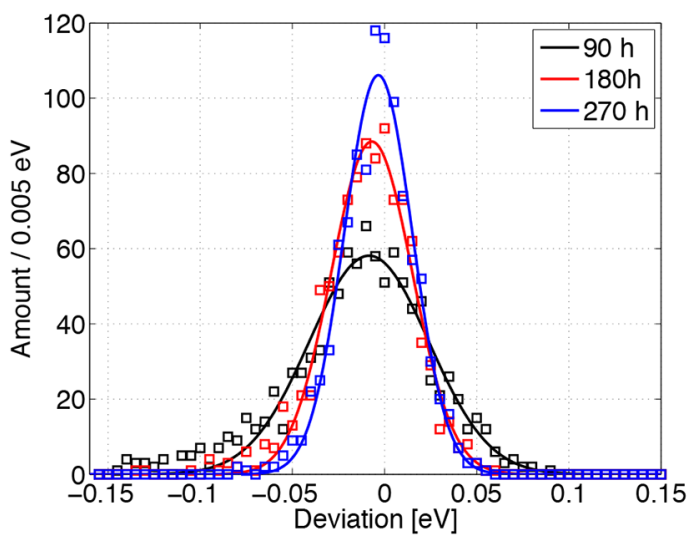

Fig. 5. Precision and accuracy of the fit method. The deviation from the simulated energy value for 1000 measurements, with different measurement times (90 h (black), $180 \mathrm{~h}$ (red), $270 \mathrm{~h}$ (blue)) is shown. The results for the corresponding Gaussian fits are shown in table 2 .

Table 2. Fit results for the function $f(x)=a \cdot \exp (-(x-$ $\left.\mu)^{2} /\left(2 \sigma^{2}\right)\right)$ fitted to the curves shown in fig. 5 .

\begin{tabular}{l|cc}
\hline Meas. time $[\mathrm{h}]$ & $\sigma[\mathrm{meV}]$ & $\mu[\mathrm{meV}]$ \\
\hline 90 & 33 & -8 \\
180 & 22 & -7 \\
270 & 18 & -3 \\
\hline
\end{tabular}

surement time $(90 \mathrm{~h}, 180 \mathrm{~h}, 270 \mathrm{~h}), 1.5 \mathrm{~V}$ blocking voltage range and blocking voltage increment of $0.04 \mathrm{~V}$ ). The fit results of the maximum kinetic energy value were then subtracted from the simulated energy and the difference was filled in a histogram. The histograms and corresponding Gaussian fits ${ }^{2}$ are plotted in fig. 5. The fit results are shown in table 2. Taking these results, it is obvious that

\footnotetext{
${ }^{2} f(x)=a \cdot \exp \left(-(x-\mu)^{2} /\left(2 \sigma^{2}\right)\right)$ was used for the fit function.
} 
the precision and accuracy both improve with longer measurement times. The width $\sigma$ follows a $\sqrt{N}$ law (starting below $50 \mathrm{meV}$ for a measurement time of $90 \mathrm{~h}$ ). Although it is much smaller than the width of the distribution, there is a shift towards lower energies, which is decreasing with better statistics. This is understood as follows. In this integrated measurement mode, the counts decrease for higher blocking voltages. The point that determines the maximum energy is also the point with the least counts. For low statistics, the counts in this point may not rise above the background, therefore the energy value that is obtained is shifted to lower energies for shorter measurement times.

\section{Conclusion and outlook}

We presented a way to measure the excitation energy of the isomeric first excited state in ${ }^{229} \mathrm{Th}$ via internal conversion electrons. The approach uses a metallic catcher to neutralize ${ }^{229 m}$ Th ions to open the IC decay channel. The analysis of simulated data results in uncertainties of below $0.1 \mathrm{eV}$ in a reasonable measurement time of $180 \mathrm{~h}(\hat{=} 7.5 \mathrm{~d})$. For longer measurement times only an incremental and asymptotically decreasing improvement can be expected.

A specific strength of this method is, that there is no influence of the sample material on the absolute achieved energy value, since only the maximum kinetic energies of the electrons are measured and no specific binding energies of electrons in the sample. Therefore, the cleanliness of the sample surface does not affect the energy measurements. Still different metallic materials can be probed to enhance confidence in the obtained energy value and investigate systematic shifts. For energies much larger than $7.8 \mathrm{eV}$, the count rate in the Fermi edge decreases. Therefore, for much larger energies and with such a low count rate, this method can only give a new lower bound for the isomeric energy. Taking all together, we conclude that it is possible to measure the isomeric energy within the currently expected energy region to better than $0.1 \mathrm{eV}$ with the proposed method.

We acknowledge fruitful discussions with S. Stellmer, M. Laatiaoui, P. Feulner, G. Dedes and J. Crespo López-Urrutia. This work was supported by DFG grant (Th956/3-1), via the European Union's Horizon 2020 research and innovation programme under grant agreement No. 664732 "nuClock" and by the LMU department of Medical Physics via the Maier-Leibnitz Laboratory.
Open Access This is an open access article distributed under the terms of the Creative Commons Attribution License (http://creativecommons.org/licenses/by/4.0), which permits unrestricted use, distribution, and reproduction in any medium, provided the original work is properly cited.

\section{References}

1. B.R. Beck et al., Phys. Rev. Lett. 98, 142501 (2007).

2. B.R. Beck et al., Proceedings of the 12th International Conference on Nuclear Reaction Mechanisms, Varenna, 2009, edited by F. Cerutti, A. Ferrari (CERN, 2010).

3. E. Peik, Chr. Tamm, Europhys. Lett. 61, 181 (2003).

4. C.J. Campbell et al., Phys. Rev. Lett. 108, 120802 (2012).

5. L.v.d. Wense et al., Nature 533, 47 (2016).

6. J. Jeet et al., Phys. Rev. Lett. 114, 253001 (2015).

7. S. Stellmer et al., Phys. Rev. C 94, 014302 (2016).

8. S.G. Porsev, V.V. Flambaum, E. Peik, Chr. Tamm, Phys. Rev. Lett. 105, 182501 (2010).

9. A. Yamaguchi et al., New J. Phys. 17, 053053 (2015).

10. G. Kazakov et al., Nucl. Instrum. Methods Phys. Res. A 735, 229 (2014).

11. C.J. Campbell et al., Phys. Rev. Lett. 106, 223001 (2011).

12. L.v.d. Wense, P.G. Thirolf, D. Kalb, M. Laatiaoui, J. Instrum. 8, P03005 (2013).

13. V. Sonnenschein et al., Eur. Phys. J. A 48, 52 (2012).

14. L.A. Kroger, C.W. Reich, Nucl. Phys. A 259, 29 (1976).

15. C.W. Reich, R.G. Helmer, Phys. Rev. Lett. 64, 271 (1990).

16. C.W. Reich, R.G. Helmer, Phys. Rev. C 49, 1845 (1994).

17. E.V. Tkalya et al., Phys. Rev. C 61, 064308 (2000).

18. F.F. Karpeshin, M.B. Trzhaskovskaya, Phys. Rev. C 76, 054313 (2007).

19. B. Seiferle, L.v.d. Wense, M. Laatiaoui, P.G. Thirolf, Eur. Phys. J. D 70, 58 (2016).

20. B. Seiferle, L.v.d. Wense, P.G. Thirolf, Phys. Rev. Lett. 118, 042501 (2017).

21. S. Köhler et al., Spectrochim. Acta B 52, 717 (1997).

22. L.v.d. Wense et al., Eur. Phys. J. A 51, 29 (2015).

23. Y. Yamakita et al., Rev. Sci. Instrum. 71, 3042 (2000).

24. J.F. Ziegler, M.D. Ziegler, J.P. Biersack, Nucl. Instrum. Methods B 268, 1818 (2010).

25. M.P. Seah, W.A. Dench, Surf. Interface Anal. 1, 2 (1979).

26. H.D. Hagstrum, Phys. Rev. 96, 325 (1954).

27. H.D. Hagstrum, Phys. Rev. 104, 309 (1956).

28. Y. Harada et al., Chem. Rev. 97, 1897 (1997).

29. S. Hüfner, Photoelectron Spectroscopy: Principles and Applications, 3rd edition (Springer, Berlin, 2003).

30. M.G. Helander et al., Appl. Surf. Sci. 256, 2602 (2010).

31. L. Devroye, Non-uniform Random Variate Generation (Springer, New York, 1986). 